\title{
Indicadores de bienestar animal durante el aturdimiento de bovinos sacrificados en establecimientos Tipo Inspección Federal del noroeste de México
}

\author{
Animal welfare indicators during the stunning of bovines slaughtered in federal inspection \\ type slaughterhouses located in northwestern Mexico \\ C Pérez-Linares ${ }^{\mathrm{a}}$, F Figueroa-Saavedra ${ }^{\mathrm{a}}$, A Estrada-Angulo ${ }^{\mathrm{b}}$, E Sánchez-López ${ }^{\mathrm{a}}$, \\ A Barreras-Serrano ${ }^{a}$, JL Bolado-Sarabia ${ }^{a}$, FG Ríos-Rincón ${ }^{b^{*}}$ \\ anstituto de Investigaciones en Ciencias Veterinarias, Universidad Autónoma de Baja California, Baja California, México. \\ ${ }^{b}$ Facultad de Medicina Veterinaria y Zootecnia, Universidad Autónoma de Sinaloa, Sinaloa, México.
}

\begin{abstract}
SUMMARY
To evaluate animal welfare indicators during stunning of bovines slaughtered in three federal inspection type slaughterhouses (A, B and C) six random visits were done, so each plant was visited twice. The stunning method was evaluated using two types of indicators, the first set was registered immediately after stunning, and the second set were measured on the severed head and considered the precision of the shot. A frequency analysis showed that $12.3 \%$ of the animals in abattoir A vocalized while the percentage in abattoir B was $31.3 \%$. Corneal reflex average in the abattoirs was lower than $3 \%$; rhythmic breathing was not seen in more than $2.2 \%$ of the cattle and between 21.1 and $34.7 \%$ of them attempted to raise the head after stunning. In abattoirs A (99.1\%), B (97.9\%) and C (96.7\%) of the bovines were stunned with just one shot. It was found that in $91.1 \%$ of the cases in abattoir A the shot was inside a radius of $2 \mathrm{~cm}$, while in abattoirs B and C the percentages were 62.8 and $70.4 \%$. In abattoir A in $41.6 \%$ of the cases the time between stunning and exsanguination was less than a minute while in abattoirs B and C the percentages were 0.9 and 27.8 . It is concluded that animal welfare indicators show shortcomings compared to international standards negatively affect and induce a high risk to the cattle welfare in the pre-mortem procedures in the stunning box and during bleeding.
\end{abstract}

Key words: stunning, bovine, animal welfare.

\section{RESUMEN}

Para evaluar indicadores de bienestar animal durante el aturdimiento de bovinos procesados en tres plantas de matanza TIF (A, B y C) se realizaron seis visitas aleatorias, dos visitas por cada planta. Se evaluó la efectividad del aturdimiento sobre indicadores de bienestar animal y de la eficacia del disparo: precisión, trayectoria y profundidad en las cabezas post mortem. Las variables se analizaron mediante frecuencias. En la planta A (12,3\%) y en la planta B (31,3\%) se registraron vocalizaciones. El promedio de reflejo corneal en los establecimientos no superó al 3\%, la respiración rítmica fue inferior al 2,2\%; el rango de intentos para levantar la cabeza fue 21,1 a 34,7\% en las tres plantas. La proporción de animales que cayeron al primer disparo fue de $99,1 \%$ (A); $97,9 \%$ (B) y 96,7\% (C), respectivamente. En la planta A, la precisión del disparo dentro del radio de 0 a $2 \mathrm{~cm}$ del blanco ideal fue de $91,1 \%$, planta B $(62,8 \%)$ y planta C (70,4\%). El intervalo entre aturdimiento y desangrado inferior a un minuto fue observado en $41,6 \%$ (A); $0,09 \%$ (B) y $27,8 \%$ (C), respectivamente. Se concluye que los indicadores de bienestar animal en los bovinos sacrificados en los establecimientos Tipo Inspección Federal del noroeste de México muestran deficiencias que, comparados con los estándares internacionales, impactan de manera negativa e inducen un alto riesgo para el bienestar de los bovinos en los procedimientos premortem, en el cajón de aturdimiento y durante el desangrado.

Palabras clave: insensibilización, bovinos, bienestar animal.

\section{INTRODUCCIÓN}

En los últimos años se ha detectado una creciente preocupación por el bienestar animal en general y en especial en los animales de producción (Aluja 2011). En México se ha despertado gran interés y preocupación por conocer si los establecimientos de matanza Tipo Inspección Federal (TIF) del país cumplen con determinados protocolos de bienestar animal exigidos por los compradores de carne del propio país así como por los importadores de Europa,

Aceptado: 12.03.2015.

* fgrios@uas.edu.mx
Estados Unidos y Canadá; estas plantas TIF están reguladas y certificadas de acuerdo con los lineamientos de la Secretaría de Agricultura, Ganadería, Desarrollo Rural, Pesca y Alimentación (SAGARPA), operan con infraestructura y equipamiento moderno, garantizan la inocuidad del producto, lo que les permite abastecer a grandes centros urbanos nacionales y exportar bajo la normativa federal (Leyva-García y col 2012).

Un aspecto relevante donde se puede afectar el bienestar en los animales de abasto es durante el aturdimiento, cuyo objetivo primordial es lograr que el animal no sienta dolor, se inmovilice y sea fácil para el operador manejarlo durante el izamiento y posterior desangrado, así como evitar efectos negativos sobre la calidad de la carne (Gallo 
y Tadich 2004). Un mal aturdimiento así como el tiempo prolongado entre este y el desangrado pueden provocar, además de sufrimiento en los animales, hemorragias detectables en los músculos de mayor valor económico (Gallo y Tadich 2004). La OIE (2009) recomienda los métodos mecánicos para el sacrificio de bovinos; estos incluyen al perno cautivo no penetrante y al perno cautivo penetrante, siendo este último el que más se usa en las plantas de sacrificio Tipo Inspección Federal en México. Gallo (2010) menciona que los indicadores más utilizados en forma práctica durante el aturdimiento son los de comportamiento: vocalizaciones, intentos de incorporarse, respiración rítmica, reflejo corneal, entre otras. El objetivo del estudio fue evaluar el efecto del aturdimiento en los indicadores de bienestar animal en bovinos procesados en tres plantas de matanza Tipo Inspección Federal ubicados en la región noroeste de México.

\section{MATERIAL Y MÉTODOS}

El estudio se llevó a cabo en tres establecimientos Tipo Inspección Federal (TIF) ubicados en la región noroeste de México (TIF A, TIF B y TIF C). Las plantas de sacrificio TIF A, B, y C tienen capacidad de sacrificar entre 350 y 400 bovinos por día en un rango que varía de 40 a 45 animales por hora, con un intervalo promedio de 1,3 minutos entre animal aturdido. En las tres plantas TIF el acceso al cajón de aturdimiento (1,80 $\mathrm{m}$ alto $\mathrm{x}$ 0,88 $\mathrm{m}$ de ancho x 2,53 $\mathrm{m}$ de largo) es por medio de una puerta tipo guillotina y con una puerta de salida de abertura horizontal. Solo la planta TIF A cuenta con un sistema de fijación de cabeza. En cada uno de los establecimientos de sacrificio el aturdimiento es realizado solo por un operador que está capacitado, y cuando este se ausenta por determinada razón, es sustituido por un operador con poca o nula experiencia. Cada planta utiliza una pistola de proyectil retenido penetrante de funcionamiento neumático (modelo USSS-1 JARVIS ${ }^{\circ}$ Jarvis Products Corporation; Middletown, CT, USA); después del aturdimiento, los bovinos son elevados al riel de desangrado mediante un pial metálico colocado en uno de los miembros posteriores; después del desangrado se transfieren a la línea de producción para continuar con el proceso de obtención de la canal, que concluye con el ingreso a las cámaras de refrigeración después de 35 a 45 minutos tras el aturdimiento.

Se registró la efectividad del aturdimiento mediante los indicadores de retorno a la sensibilidad basados en la metodología propuesta por Grandin (2012), aplicada inmediatamente después de efectuado el disparo en la cabeza de los bovinos. Se evaluaron los indicadores de bienestar animal, la regurgitación de contenido ruminal por la vía oral-nasal; la precisión del disparo en la región frontal del cráneo, mediante plantilla transparente (HSA 1995); distancia (en cm) desde el centro del blanco de la plantilla hacia el orificio donde ingresó el perno cautivo después del disparo; esta distancia se clasificó en cuatro categorías numéricas (de 0 a $2 \mathrm{~cm}$; de 2,1 a $4 \mathrm{~cm}$; de 4,1 a $6 \mathrm{~cm}$; de 6,1 a $8 \mathrm{~cm}$ ); la orientación del disparo clasificada basado en los puntos cardinales (NO, NE, SO y $\mathrm{SE})$; la trayectoria fue evaluada de acuerdo con la forma en cómo ingresó el disparo (perpendicular a la superficie ósea y diagonal); la profundidad del disparo en el cráneo se midió con un tubo de silicón graduado en cm, que se introdujo en el orificio que produjo en el cráneo el perno del equipo aturdidor. En los bovinos aturdidos en la nuca no se utilizó la plantilla transparente, solo se registraron los indicadores de retorno a la sensibilidad posterior a la ejecución del disparo. Las variables en estudio se analizaron mediante el procedimiento FREQ del paquete SAS (SAS 2001).

\section{RESULTADOS Y DISCUSIÓN}

Los resultados de la evaluación de indicadores del bienestar animal tras el aturdimiento se muestran en el cuadro 1. La frecuencia de bovinos que vocalizaron después del disparo en dos de las tres plantas fueron la planta TIF A con el $12,3 \%$ y la planta TIF B con $31,3 \%$. Una de las variables indicadoras de estrés es la vocalización debida a un aturdimiento deficiente (Warriss y col 1984), además es un indicador del bienestar animal, y como es de fácil cuantificación es posible identificar fallas en el equipo aturdidor o de manejo inapropiado (Manteuffel y col 2004); en el presente estudio se observó que los porcentajes en dos de las tres plantas son más bajos a los que reportan Gallo y col (2003) con 46,9\% de bovinos que vocalizaron antes de cambiar la infraestructura y capacitar a los operarios; mientras que otros estudios realizados en Estados Unidos de América y en Chile, respectivamente, refieren porcentajes de 3,08\% (Grandin 2005) y 0,3\% (Concha 2010).

En las tres plantas TIF se observó que el promedio del reflejo corneal no superó al 3,0\% con relación al número de bovinos aturdidos, mientras que el promedio del indicador de respiración rítmica no fue mayor al 2,2\%. La frecuencia de reflejo corneal observada en este estudio es similar a la que mencionan Cartes (2000) con 2,6\%, pero inferior a la registrada por Concha (2010) con 4,9\% y Gallo y col (2003) con 66,9\%. Grandin (1994) refiere que un buen indicador de inconciencia en el ganado bovino después del aturdimiento es la no presentación de reflejo corneal, por lo que la presentación de este signo es un indicador de una posible recuperación de la sensibilidad.

La presencia del pataleo estuvo presente en valores de 35,$7 ; 39,5$ y 71,9\% en las plantas TIF C, B y A, respectivamente, en tanto que se observó un rango entre 21,2 a $34,7 \%$ de bovinos que presentaron intentos de levantar la cabeza. Respecto de la sensibilidad al corte de cuernos solo en la planta TIF A se observó en el 2,0\% en los animales sacrificados, esto quizás porque la actividad se lleva a cabo antes del desangrado, lo que indica que estos bovinos no estuvieron debidamente aturdidos. 
Cuadro 1. Signos indicadores de retorno a la sensibilidad durante el proceso de aturdimiento de los bovinos en tres plantas Tipo Inspección Federal. Sensibility signs after stunning of cattle in three Type Federally Inspected abattoirs.

\begin{tabular}{|c|c|c|c|c|c|c|}
\hline \multirow[b]{2}{*}{ Signos de retorno a la sensibilidad } & \multicolumn{2}{|c|}{$\begin{array}{c}\text { Planta TIF A } \\
n=463\end{array}$} & \multicolumn{2}{|c|}{$\begin{array}{l}\text { Planta TIF B } \\
n=182\end{array}$} & \multicolumn{2}{|c|}{$\begin{array}{c}\text { Planta TIF C } \\
n=406\end{array}$} \\
\hline & Presencia & Ausencia & Presencia & Ausencia & Presencia & Ausencia \\
\hline Vocalización & $57(12,3 \%)$ & 406 & $57(31,3 \%)$ & 125 & - & - \\
\hline Reflejo corneal & $26(5,6 \%)$ & 437 & $2(1,09 \%)$ & 180 & $8(1,97 \%)$ & 398 \\
\hline Respiración rítmica & - & - & $5(2,74 \%)$ & 177 & $6(1,47 \%)$ & 400 \\
\hline $\begin{array}{l}\text { Movimiento de extremidades } \\
\text { (Pataleo clónico) }\end{array}$ & 333 & 130 & $72(39,5 \%)$ & 90 & $145(35,7 \%)$ & 261 \\
\hline Intento de levantar la cabeza & $161(34,7 \%)$ & 302 & $53(29,1 \%)$ & 129 & $86(21,2 \%)$ & 320 \\
\hline Movimiento de cola & $196(42,3 \%)$ & 267 & $36(19,8 \%)$ & 146 & $48(11,8 \%)$ & 358 \\
\hline Sensibilidad al corte de cuernos & $12(2,6 \%)$ & 451 & 0 & 182 & 0 & 406 \\
\hline $\begin{array}{l}\text { Regurgitación del contenido } \\
\text { ruminal }\end{array}$ & 0 & 463 & $4(2,2 \%)$ & 178 & $14(3,5 \%)$ & 392 \\
\hline
\end{tabular}

En el presente estudio la frecuencia registrada en respiración rítmica está muy por debajo de las que refieren Cartes (2000) con 4,1\%; y Concha (2010) con 6,3\%. Blackmore y Delany (1988) mencionan que la respiración rítmica cesa después de haber efectuado el disparo. Gracey y col (1999) consideran la ausencia de la respiración rítmica como signo constante de pérdida de conocimiento.

Respecto de los resultados de intento de levantar la cabeza, en este estudio fue mayor al registrado por Gallo y Cartes (2000) donde reportan 19,8\%; estos valores superan al referido por Concha (2010) con el 0,9\% de los animales sacrificados. En este sentido, Dalmau y col (2012) mencionan que además de los signos de un mal aturdimiento como la presencia de vocalizaciones y la reaparición del ritmo respiratorio, también se presentan intentos de incorporarse.

En la planta TIF B y TIF C se registraron bovinos que expulsaron contenido ruminal por la vía oral (4 y 14 bovinos, respectivamente) al momento del izado. Con referencia a la expulsión del contenido ruminal, Ríos-Rincón y col (2012) mencionan que algún fallo operacional durante el intervalo aturdimiento-desangrado prolonga la agonía del bovino con un gran esfuerzo respiratorio provocado por un enfisema pulmonar a consecuencia de una inspiración forzada y jadeante observada comúnmente en bovinos aturdidos de manera incorrecta en la planta de sacrificio, lo que provoca la expulsión mecánica del contenido ruminal por las vías oral y nasal; por tanto esta lesión es un posible indicador de un pobre bienestar animal durante el desangrado.

Grandin (2002) menciona que debe haber tolerancia cero para iniciar cualquier procedimiento de sacrificio después del desangrado como remover patas, cuernos y cabeza en un animal que muestre signos de retorno a la sensibilidad.
Por tal motivo, el aturdimiento de los animales de abasto tiene el propósito de evitarles sufrimiento innecesario al momento de provocarles la muerte; esta es una operación obligatoria de acuerdo con los estándares de bienestar animal establecidos por la OIE (2008), y al mismo tiempo indica que las fallas más frecuentes se deben a la falta de puntería, a la velocidad y al diámetro del perno cautivo, además de que los operarios deben ser competentes en el manejo y mantenimiento del equipo aturdidor y tener experiencia en su operación.

Los resultados de las otras variables evaluadas tras el aturdimiento se muestran en el cuadro 2. Respecto del número de animales noqueados al primer disparo la planta TIF A registró 99,1\% mientras que en las plantas TIF B y TIF C se observaron $97,9 \%$ y $96,7 \%$ de los bovinos que cayeron al primer disparo, respectivamente. En referencia a la evaluación después del disparo, Grandin (2000) la califica como de grado excelente cuando el 99 a $100 \%$ de los animales caen al primer disparo; en esta calificación se ubica la planta TIF A, mientras que las plantas TIF B y TIF C se ubican en la categoría aceptable (95 al 98\% de los bovinos que caen al primer disparo). El porcentaje de bovinos que fueron aturdidos al primer disparo en cada uno de los tres establecimientos objeto del presente estudio superan a los que se obtuvieron en otras investigaciones, por ejemplo, Concha (2010) con 86,2\%, y Gallo y Cartes (2000) con $83,6 \%$. Entre los factores que provocan se efectúe más de un disparo para lograr efectividad en el aturdimiento está la posición incorrecta de la pistola en la parte frontal del cráneo, la insuficiente fuerza que impulsa al pistón cautivo, así como el uso de un cartucho inadecuado para el tamaño del animal (HSA 2006).

Al evaluar la precisión del disparo en la posición correcta, en la planta TIF A se registró el valor más alto, 
Cuadro 2. Resultados de las variables evaluadas en las cabezas post mortem de bovinos y el tiempo entre disparo y desangrado. Results of the variables evaluated in post-mortem bovine heads and the time between shot and bleeding.

\begin{tabular}{|c|c|c|c|}
\hline \multirow{2}{*}{ Variable evaluada } & \multicolumn{3}{|c|}{ Planta de sacrificio } \\
\hline & TIF A $n=463$ & TIF B $n=194$ & TIF C $n=406$ \\
\hline \multicolumn{4}{|l|}{$\mathrm{N}^{\circ}$ de disparos } \\
\hline 1 & 459 & 190 & 393 \\
\hline 2 & 4 & 4 & 11 \\
\hline 3 & & & 1 \\
\hline 4 & & & 1 \\
\hline Precisión (cm) & $n=284$ & $\mathrm{n}=194$ & $\mathrm{n}=183$ \\
\hline $0-2$ & 259 & 122 & 129 \\
\hline $2,1-4$ & 24 & 60 & 46 \\
\hline $4,1-6$ & 1 & 10 & 6 \\
\hline Nuca & & 2 & 2 \\
\hline Orientación & $\mathrm{n}=284$ & $\mathrm{n}=194$ & $\mathrm{n}=183$ \\
\hline Centro & 100 & 6 & \\
\hline NE & 59 & 60 & 31 \\
\hline NO & 16 & 78 & 73 \\
\hline SE & 66 & 19 & 15 \\
\hline SO & 43 & 31 & 64 \\
\hline Trayectoria & $\mathrm{n}=284$ & $\mathrm{n}=194$ & $\mathrm{n}=183$ \\
\hline Diagonal & 66 & 65 & 75 \\
\hline Perpendicular al cráneo & 218 & 129 & 108 \\
\hline Profundidad del disparo & $\mathrm{n}=284$ & $\mathrm{n}=194$ & $\mathrm{n}=183$ \\
\hline $0-2,5 \mathrm{~cm}$ & 7 & 4 & 4 \\
\hline $2,5-5,0 \mathrm{~cm}$ & 46 & 28 & 16 \\
\hline $5,0 \mathrm{~cm}-7,5 \mathrm{~cm}$ & 149 & 113 & 129 \\
\hline$>7,5 \mathrm{~cm}$ & 82 & 49 & 36 \\
\hline Intervalo entre aturdimiento y desangrado & $\mathrm{n}=463$ & $\mathrm{n}=182$ & $\mathrm{n}=406$ \\
\hline Hasta $30 \mathrm{seg}$ & 27 & 2 & 32 \\
\hline 31 a $60 \mathrm{seg}$ & 166 & 15 & 81 \\
\hline Mayor a $60 \mathrm{seg}$ & 270 & 165 & 293 \\
\hline
\end{tabular}

con $91,1 \%(259 / 284)$ de los bovinos que presentaron la región del disparo dentro de un radio de 0 a $2 \mathrm{~cm}$ respecto del blanco ideal, mientras que en la planta TIF B y C se observaron el $62,8 \%(122 / 194)$ y $70,4 \%$ (129/183), respectivamente. De acuerdo con la orientación del disparo se observó que en la planta TIF A el 35\% de los disparos fueron certeros en el centro del cráneo, mientras que en las otras dos plantas TIF los disparos se distribuyeron en los cuatro puntos cardinales. En lo que se refiere a la trayectoria, el rango varía de 59 al $76 \%$ del disparo realizado en forma perpendicular a la superficie frontal del cráneo en las tres plantas TIF. De acuerdo con la profundidad del disparo en el cráneo bovino se observó un rango de 5,0 a $7,5 \mathrm{~cm}$ en las tres plantas de proceso objeto del presente estudio. En relación con el intervalo entre el aturdimiento y el desangrado la planta TIF A mostró el mayor porcentaje de bovinos desangrados antes de un minuto $(41,6 \%)$ en comparación con los porcentajes observados en las plantas TIF B y TIF C (con $0,09 \%$ y $27,8 \%$, respectivamente).

El origen de la falla de un aturdimiento efectivo es la funcionalidad del cajón del noqueo, ya que este puede llegar a ser demasiado ancho para los animales de menor tamaño (vaquillas y novillos), lo que permite que estos animales se agiten, se giren y con ello, el resultado del disparo sea incorrecto. Estos resultados han sido referidos por Muñoz y col (2012) y son similares a los que se observaron en las plantas TIF B y TIF C donde el comportamiento excitable del animal dentro del cajón de noqueo exige usar con regular frecuencia la picana eléctrica para acomodarlos y por consecuencia una mayor renuencia del bovino; con 
esta acción se manifiesta la mayor vocalización del ganado e incrementa la dificultad para lograr un disparo certero.

En relación con la precisión del disparo, la diferencia observada entre la planta TIF A respecto de las otras dos puede ser atribuida a que en esa planta TIF se cuenta con sujetador de cabeza; la existencia de un sistema de sujeción para los bovinos en el cajón de aturdimiento ayuda a mejorar la efectividad del proceso (Gallo y col 2003), porque facilita al operador ejecutar con precisión el disparo y este sea más certero en la cabeza del bovino. OIE (2011) recomienda insensibilizar al animal sin demora alguna una vez aplicado el dispositivo para mantener sujeto al bovino durante pocos segundos, para garantizar su eficacia y reducir el efecto estresante en el ganado. Gregory (1998) menciona que cuando el disparo se desvía por más de $2 \mathrm{~cm}$ de su posición ideal, existe una asociación con un aturdimiento imperfecto y pueden manifestarse signos de conciencia, comprometiendo con ello el bienestar del animal. Para aturdir correctamente a los animales de abasto Miranda de la Lama (2013) menciona que se requiere una combinación de destreza y entrenamiento del operario.

Acerca de los resultados observados en las tres plantas TIF concernidos con la trayectoria perpendicular del disparo en relación al hueso frontal del cráneo se debe a que al operador se le facilita ejecutar la acción de esta manera, debido al movimiento de cabeza del bovino que está alojado en el cajón de aturdimiento. En este sentido, es importante considerar el diseño apropiado del cajón de aturdimiento para que el operador pueda con mayor facilidad colocar de forma correcta el equipo aturdidor y efectuar el disparo con precisión; en referencia a lo anterior, la OIE (2009) establece que para producir inconciencia efectiva el equipo debe ser colocado correctamente sobre la parte frontal media de la cabeza del bovino.

HSA (2006) menciona que el intervalo entre el disparo y desangrado debe mantenerse al mínimo, siendo aceptado los intervalos máximos de 60 segundos al utilizar el sistema de perno cautivo con penetración. En tal sentido, Cáraves y col (2006) condujeron un estudio en 6 plantas de sacrificio y observaron que el intervalo aturdimiento-desangrado mayor a 60 segundos ocurrió en el 36,8\% de animales; en un estudio conducido por Concha (2010) se observó que en el 81,4\% de los bovinos el intervalo fue superior a 60 segundos y en el 17,1\% fue menor de 60 segundos.

OIE (2011) recomienda que en atención al bienestar, los animales que hayan sido aturdidos con un método reversible deberán ser sometidos al proceso de sangrado sin dilación; cuando el bovino es aturdido con perno cautivo penetrante el plazo máximo para iniciar el sangrado que no deberá demorarse más de 30 segundos y contribuir a la mejora de un desangrado eficaz (Gregory 2005).

La evaluación de los indicadores de bienestar animal en los bovinos sacrificados en los establecimientos Tipo Inspección Federal del noroeste de México muestran deficiencias que, comparados con los estándares internacionales, impactan de manera negativa e inducen un alto riesgo para el bienestar de los bovinos en los procedimientos pre mortem, en el cajón de aturdimiento. La efectividad en la aplicación del disparo y la consiguiente mejora de los indicadores de bienestar animal en los establecimientos se pudiese optimizar si se realiza adecuada y oportunamente un programa de mantenimiento preventivo del equipo, además de promover un programa de capacitación al personal que realiza esta labor, sin descuidar los aspectos relacionados con la supervisión y la creación de un programa de incentivos económicos; esto se verá reflejado en el aseguramiento del bienestar animal, la mejora de la calidad del manejo de los bovinos y en la interacción humano-animal en la planta de proceso.

\section{AGRADECIMIENTOS}

Al personal que labora en las plantas de sacrificio TIF de la ciudad de Mexicali, Baja California y Culiacán, Sinaloa, México, por las facilidades otorgadas para la realización de esta investigación.

\section{REFERENCIAS}

Aluja AS. 2011. Animal welfare in the Veterinary Medicine and animal husbandry curriculum. What for and why. Vet Mex 42,137-147.

Blackmore D, M Delany. 1988. Slaughter of stock. Chapter 4: Assessment of insensibility. Department of Veterinary Pathology and Public Health, Masey University. Publication No. 118, Pp 23-27.

Cáraves M, C Gallo, A Strappini, L Aguayo, A Barrientos, R Allende, F Alarcón, I Briones. 2006. Evaluación del bienestar animal de bovinos durante el manejo antemortem en seis plantas de faenado en Chile. Libro de resúmenes XXXI de la Sociedad Chilena de Producción Animal, Pp 79-80.

Cartes MM. 2000. Evaluación de la eficacia en el uso de la pistola de proyectil retenido para insensibilizar ganado bovino en tres plantas faenadoras de carne de la Décima Región. Tesis, Facultad de Medicina Veterinaria, Universidad Austral de Chile, Valdivia, Chile.

Concha RA. 2010. Evaluación de la eficacia en el uso de la pistola de proyectil retenido sin penetración de cráneo para insensibilizar ganado bovino en una planta faenadora de carne. Tesis, Facultad de Medicina Veterinaria, Universidad Austral de Chile, Valdivia, Chile.

Dalmau A, P Llonch, A Velarde. 2012. Sacrificio religioso de animales para consumo. En: Mota RD, Huertas SM, Guerrero I, Trujillo ME (eds). Bienestar Animal. Elsevier, México, Pp 446.

Gallo C, M Cartes. 2000. Insensibilización en bovinos: evaluación de la eficacia de la pistola de proyectil retenido en tres plantas de la Décima Región. XII Congreso de Medicina Veterinaria, Universidad de Chile, Santiago, Chile.

Gallo C, M Teuber, M Cartes, H Uribe, T Grandin. 2003. Improvements in stunning of cattle with a pneumatic stunner after changes in equipment and employee training. Arch Med Vet 35,159-170.

Gallo C, N Tadich. 2004. Bienestar animal y calidad de la carne durante los manejos previos al faenamiento en bovinos. En: Resúmenes Seminario "Producción animal de calidad contemplando el bienestar animal”, Universidad Austral de Chile, Valdivia, Chile, Pp. 41-56.

Gallo C. 2010. Bienestar animal y buenas prácticas de manejo relacionadas con la calidad de la carne. En: Bianchi G, Feed $\mathrm{OD}$ (eds). Introducción a la ciencia de la carne. $1^{\mathrm{a}}$ ed. Editorial Hemisferio Sur, Buenos Aires, Argentina, Pp 455-494.

Gracey JF, DS Collins, RJ Huey. 1999. Humane slaughter. In: Meat hygiene. $10^{\text {th }}$ ed. WB Saunders Company LTD, London, UK, Pp 197-222.

Grandin T. 1998. Objective scoring of animal holding and stunning practices at slaughter plants. J Am Vet Med Assoc 212, 36-39. 
Grandin T. 2000. Effect of animal welfare audits of slaughter plants by a major fast food company on cattle handling and stunning practices. J Am Vet Med Assoc 216,848-851.

Grandin T. 2002. Return sensibility problems after penetrating captive bolt stunning of cattle in comercial beef slaughter plants. J Am Vet Med Assoc 221, 1258-1261.

Grandin T. 2005. Maintenance of good animal welfare standards in beef slaughter plants by use of auditing programs. J Am Vet Med Assoc 226, 370-373.

Grandin T. 2012. Recommended Animal Handling Guidelines \& Audit: A Systematic Approach to Animal Welfare. American Meat Institute, Washington DC, USA.

Gregory NG. 1998. Stunning and meat quality. In: Gregory NG, Grandin T. Animal welfare and meat quality. CABI Publishing, New York, USA, Pp 241-253.

Gregory NG. 2005. Recent concerns about stunning and slaughter. Review. Meat Sci 70,481-491.

HSA, Humane Slaughter Association. 1995. Unit 10: Using the captive bolt. Wheathampstead, UK, Pp 53-58.

HSA, Humane Slaughter Association. 2006. Insensibilización de Ganado con pistola neumática de perno cautivo. Wheathampstead, UK, Pp 3-13.

Leyva-García IA, F Figueroa-Saavedra, E Sánchez-López, C PérezLinares, A Barreras-Serrano. 2012. Impacto económico de la presencia de carne DFD en una planta Tipo Inspección Federal. Arch Med Vet 44, 39-42.
Manteuffel G, B Puppe, P Schön. 2004. Vocalization of farm animal as a measure of welfare. Appl Anim Behav Sci 88, 163-182.

Miranda de la Lama GC. 2013. Transporte y logística presacrificio: principios y tendencias en bienestar animal y su relación con la calidad de la carne. Vet Méx 44, 31-56.

Muñoz D, A Strappini, C Gallo. 2012. Indicadores de bienestar animal para detectar problemas en cajón de insensibilización en bovinos. Arch Med Vet 44, 297-302.

OIE, Organización Mundial de Sanidad Animal. 2009. Normas Sanitarias. Código Sanitario para Animales Terrestres. Directrices para el sacrificio de animales destinados al consumo humano. Parte 3, Título 3.7, Anexo 3.7.5.

OIE. Organización Mundial de Sanidad Animal. 2011. Código Sanitario para los animales terrestres. Directrices para el sacrificio de animales destinados al consumo humano. Editorial OIE, París, Francia.

Ríos-Rincón FG, A Estrada-Angulo, J Hernández-Bautista, C PérezLinares, JJ Portillo-Loera y JC Robles-Estrada. 2012. Factores que influyen en la emesis postaturdimiento en bovinos. Rev Mex Cienc Реси 3, 343-356.

Warriss PD, C. Kestin, N Brown, J Wilkins. 1984. The time required for recovery from mixing stress in young bulls and the prevention of dark cutting meat. Meat Sci 10, 53-68.

SAS. 2001. AS/STAT 9.1 User's Guide. Volumes 1-7. SAS Inst., Cary, N. C., USA. 